\title{
Low prevalence of Pneumocystis jirovecii lung colonization in Ugandan HIV-infected patients hospitalized with non- Pneumocystis pneumonia
}

Steve M Taylor, MD, MPH ${ }^{1,2,{ }^{*}}$, Steven R Meshnick, MD, $\mathrm{PhD}^{1}$, William Worodria, MBChB ${ }^{3,4}$, Alfred Andama, BSc ${ }^{3,4}$, J. Lucian Davis, MD, MAS ${ }^{3,5}$, Adithya Cattamanchi, MD, MAS ${ }^{3,5}$, Saskia den Boon, MSc, PhD ${ }^{3}$, Samuel D Yoo, MD ${ }^{3,4}$, Carol D. Goodman ${ }^{6}$, and Laurence Huang, MD, MAS ${ }^{3,5,7}$ On behalf of the International HIV-associated Opportunistic Pneumonias (IHOP) Study

${ }^{1}$ Department of Epidemiology, Gillings School of Global Public Health, University of North Carolina, Chapel Hill, USA ${ }^{2}$ Division of Infectious Diseases and International Health, Duke University Medical Center, Durham, NC, USA ${ }^{3}$ Makerere University-University of California, San Francisco Research Collaboration, Kampala, Uganda ${ }^{4}$ Department of Medicine, Makerere College of Health Sciences, Mulago Hospital, Kampala, Uganda ${ }^{5}$ Division of Pulmonary and Critical Care Medicine, San Francisco General Hospital, University of California, San Francisco, USA ${ }^{6}$ Department of Laboratory Medicine, San Francisco General Hospital, San Francisco, USA ${ }^{7}$ HIV/AIDS Division, San Francisco General Hospital, University of California, San Francisco, USA

\section{Abstract}

Pneumocystis jirovecii is an important opportunistic infection in HIV-infected patients. In the developed world, $P$. jirovecii epidemiology is marked by frequent colonization in immunosuppressed patients, but data on the prevalence of colonization is very limited in subSaharan Africa, where the majority of persons living with HIV reside. Our objective was to describe the epidemiology of $P$. jirovecii colonization among HIV-positive patients in a crosssectional, hospital-based study of patients admitted with suspected pneumonia in Kampala, Uganda. $P$. jirovecii was detectable in bronchoalveolar lavage fluid from 7 of $124(6 \%)$ consecutive patients with non-Pneumocystis pneumonia. Colonization was not associated with patient demographic or clinical information. This prevalence is substantially lower than in published studies in the developed world, and suggests that there is a limited reservoir of organisms for clinical infections in this Ugandan population. These findings may partially explain the low incidence of Pneumocystis pneumonia in Uganda and other sub-Saharan African countries.

(C) 2011 Elsevier Inc. All rights reserved.

*Corresponding author: Steve M Taylor, 135 Dauer Drive, MHRC 3113, Campus Box 7435, Chapel Hill, NC 27599, taylo115@email.unc.edu, Phone: 919/843-4384,FAX: 919/966-0584.

Conflict of Interest

All authors declare that they have no competing interests.

Publisher's Disclaimer: This is a PDF file of an unedited manuscript that has been accepted for publication. As a service to our customers we are providing this early version of the manuscript. The manuscript will undergo copyediting, typesetting, and review of the resulting proof before it is published in its final citable form. Please note that during the production process errors may be discovered which could affect the content, and all legal disclaimers that apply to the journal pertain. 


\section{Keywords}

Pneumocystis jirovecii; colonization; pneumonia; AIDS

\section{Introduction}

The detection of Pneumocystis jirovecii in the respiratory tract of patients without clinical or microscopic Pneumocystis pneumonia (PcP) represents colonization. Although its clinical significance is unclear, an improved understanding of Pneumocystis colonization can provide insight into the epidemiology of $\mathrm{PcP}$ in high-risk groups, including the temporal and geographic acquisition of organisms and the environmental reservoirs of $P$. jirovecii. In humans, the prevalence of Pneumocystis colonization varies between patient groups, but is most strongly associated with immunosuppression and may correlate with susceptibility to clinical PcP.

The epidemiology and burden of $P$. jirovecii infection is heterogeneous in developing countries with high prevalences of HIV such as those in sub-Saharan Africa. Serologic investigations indicate that exposure to P. jirovecii is common in South Africa, Cameroon, and the Gambia, and $P$. jirovecii is a significant cause of pneumonia and death in HIVinfected infants in southern Africa. In adults, the prevalence of PcP amongst HIV-infected patients with pneumonia has been reported between 5\% and 39\%, but these may be underestimates owing to the difficulty of microscopic diagnosis in these resource-limited settings. The widespread exposure to $P$. jirovecii early in life but relatively low incidence of infection in high-risk adults could be explained by either a limited reservoir for $P$. jirovecii transmission in adults or the acquisition of protection from clinical diseases in adults.

The epidemiology of $P$. jirovecii colonization in high-risk HIV-infected patients in subSaharan Africa is largely unknown. One study reported a prevalence of $9 \%$, although it enrolled only outpatients and relied upon detection of Pneumocystis in induced sputa, which is less sensitive than bronchoscopy. Because colonized patients serve as a reservoir for transmission, improved understanding of this phenomenon may inform estimates of the burden of clinical disease due to $P$. jirovecii. Herein, we endeavor to describe the epidemiology of $P$. jirovecii colonization among hospitalized, HIV-infected Ugandans with suspected pneumonia by applying a sensitive molecular assay to detect $P$. jirovecii DNA in bronchoalveolar lavage (BAL) specimens collected in a cross-sectional study of HIVinfected patients hospitalized with non-Pneumocystis pneumonia at Mulago Hospital, a national referral center in Kampala, Uganda.

\section{Materials and Methods}

\section{Ethics statement}

The study was approved by institutional review boards at Mulago Hospital and Makerere University, the University of California, San Francisco, the University of North Carolina at Chapel Hill, and by the Uganda National Council for Science and Technology.

\section{Enrollment and data collection}

Consecutive patients admitted to Mulago Hospital were screened for inclusion between September 2007 and October 2008. Patients were enrolled if they had cough $\geq 2$ weeks but $<6$ months, were HIV-infected, had received a clinical diagnosis of pneumonia, and provided informed consent. Clinical and demographic information were collected on standardized forms. 
All patients had a chest $\mathrm{x}$-ray performed and two sputum samples examined for acid-fast bacilli (AFB) by direct Ziehl-Neelsen microscopy; those with sputa negative for AFB were referred for bronchoscopy with BAL. Two experienced pulmonologists performed bronchoscopies, which included complete visualization of central airways and BAL with up to $125 \mathrm{~mL}$ of sterile normal saline lavaged into the lobe of the lung most affected on chest $\mathrm{x}$ ray.

BAL specimens were examined by trained laboratory technicians in the Microbiology Department at Mulago Hospital and, for mycobacterial testing, the National Tuberculosis Reference Laboratory. Analyses included AFB smear and Lowenstein-Jensen culture for $M$. tuberculosis, smear and culture for fungi, and modified Giemsa stain for $P$. jirovecii. In addition, Giemsa-stained BAL specimens were re-reviewed for $P$. jirovecii by a masked, independent reader at San Francisco General Hospital. Aliquots of $1-2 \mathrm{~mL}$ of unprocessed BAL fluid were frozen at $-20^{\circ} \mathrm{C}$ for subsequent molecular analyses.

\section{Molecular analyses}

PCR testing was performed at the University of North Carolina by personnel masked to clinical and microscopic diagnoses. BAL specimens were thawed and briefly vortexed. Genomic DNA (gDNA) was extracted from 200uL of each BAL specimen using the EZ1 Virus Mini Kit v2.0 (Qiagen, Valencia, CA). Individual samples were amplified in a nested PCR assay that targets the $P$. jirovecii mitochondrial large subunit of ribosomal DNA (mtLSUrRNA) using a standard protocol. Products were electrophoresed on $1 \%$ agarose gels. Separate work areas for gDNA extraction, reaction set- up, and electrophoresis were maintained to minimize contamination of the PCR testing, and all reaction plates included negative and positive controls with either molecular-grade water or gDNA from a patient with known PcP, respectively.

Samples demonstrating amplification were purified with the QIAquick Gel Extraction Kit (Qiagen). Purified amplicons were bi-directionally sequenced at the UNC Core Sequencing Facility using the ABI PRISM BigDye Version 1.1 Terminator Cycle Sequencing Ready Reaction Kit with AmpliTaqR DNA Polymerase (Applied Biosystems, Foster City, CA). Sequences were analyzed with Sequencher v4.10 (Gene Codes, Ann Arbor, MI) after alignment with the reference mtLSUrRNA sequence M58605.1, and genotypes were assigned according to standard nomenclature.

\section{Definitions and statistical analyses}

These analyses included patients with a negative modified Giemsa smear for PcP, originally read at Mulago Hospital and confirmed as negative at San Francisco General Hospital. $P$. jirovecii colonization was defined as the presence of $P$. jirovecii DNA, as determined by the amplification of the mtLSUrRNA target, in a PcP-negative patient. Final diagnoses were determined based on microbiologic results and clinical response. Bacterial pneumonia was assigned as a final diagnosis in the event of improvement with empiric antimicrobial therapy without a confirmed microbiologic diagnosis. Final diagnosis was categorized as "unknown" when the patient died prior to completing the diagnostic evaluation or when the evaluation was negative and any response to empiric therapy was unable to be assessed because the patient was lost to follow-up. Post-discharge vital status was assessed either in person or by telephone two months after discharge. Antiretrovirals were not routinely begun during hospitalization or at discharge; their initiation after discharge was at the treating clinician's discretion, but generally would not have been done within 2 months after discharge.

All data were analyzed using Stata/IC (version 10, Stata Corp, College Station, TX). For the cross-sectional data, we calculated means, medians, and proportions of clinical predictors in 
patients with and without colonization, and assessed bivariate associations by using Fisher's exact or the Kruskal-Wallis test for categorical or continuous variables, respectively. Using the two-month follow-up data, we calculated a risk-ratio for death either prior to or after discharge. A two-tailed $\mathrm{p}<0.05$ was considered significant for all analyses.

\section{Results}

Of 130 patients who underwent bronchoscopy, 124 had negative modified Giemsa stains and were thus PcP-negative. Median age was 33 years (interquartile range $28-39$ years) and $57 \%$ were women. Final diagnoses in these PCP-negative patients were tuberculosis (30\%), bacterial pneumonia (24\%), multiple diagnoses (23\%), and unknown (23\%). Among the 30 patients with multiple pulmonary diagnoses, the most common diagnoses were Cryptococcus neoformans (14), pulmonary tuberculosis (11), and pulmonary Kaposi's sarcoma (9), each in conjunction with other diagnoses. HIV was a new diagnosis for 31 patients (25\%). Of the 93 patients with known HIV infection at admission, 77 (83\%) reported taking PcP prophylaxis with trimethoprim-sulfamethoxazole or dapsone. Of all 124 patients, 34 patients (27\%) died either prior to discharge or within two months of discharge.

Specimens from 7 of 124 patients (6\%) were positive in the mtLSUrRNA assay, indicating colonization with $P$. jirovecii. All positive and negative controls returned expected results. There were no significant differences in clinical presenting factors between colonized and non-colonized patients (Table 1). Notably, among the 31 patients for whom HIV was a new diagnosis upon admission, 2 (7\%) were colonized. At 2-month follow-up, 71\% (5/7) colonized patients had died, compared with 25\% (29/117) of non-colonized patients (Risk Ratio 2.9; 95\% confidence interval 1.6 - 5.1).

All seven colonized patients had CD4 cell counts below 200 cells $/ \mathrm{mm}^{3}$ (range 7 - 171). Five patients were diagnosed with pulmonary tuberculosis (with or without a secondary diagnosis), one had pulmonary Kaposi's sarcoma, and in one patient the diagnosis was unknown. Five of seven patients had known HIV infection upon admission, all of whom reported taking PcP prophylaxis with cotrimoxazole prior to presentation. Genotyping of the mtLSUrRNA amplicons demonstrated genotype 1 in four patients and genotype 3 in six patients (three patients had both genotypes present concurrently).

\section{Discussion}

In this population of HIV-infected patients with non-Pneumocystis pneumonia admitted to a referral hospital in Kampala, Uganda, only $6 \%$ of patients were colonized with $P$. jirovecii. Although this may reflect the frequent receipt of PcP prophylaxis (83\%) among the patients with known HIV, even among the 31 patients in whom HIV was newly diagnosed only $7 \%$ were colonized. Additionally, although the low colonization prevalence precluded a full analysis, colonized patients were almost three times as likely to die within two months after discharge. Because colonized individuals may serve as reservoirs for $P$. jirovecii transmission, the low prevalence of colonization in these high-risk patients suggests that in Uganda, in comparison with other settings, transmission of $P$. jirovecii is limited. (Kyeyune, den Boon et al. 2010)

The overall prevalence of colonization in our cohort of HIV-infected patients is substantially lower than in other settings. Majorities of similar patients hospitalized with nonPneumocystis pneumonia in San Francisco (69\%) and New Orleans (68\%) were colonized when tested with the same molecular assay. When using a similar assay, $46 \%$ of HIVinfected men dying of non-Pneumocystis respiratory illnesses were colonized, although this varied between US cities from $16 \%$ to $70 \%$, suggesting geographic differences in the 
prevalence of $P$. jirovecii colonization. Study design differences may contribute to the variation between studies, but we believe our study accurately reflects the epidemiology of $P$. jirovecii colonization in Kampala owing to the enrollment of consecutive patients with clear inclusion criteria and the employment of a high standard of diagnostic and microbiologic testing.

Several factors may account for this low prevalence. Use of PcP prophylaxis was common in this cohort, though this has not been associated with reduced colonization in other studies. Moreover, colonization was similarly infrequent in patients without a prior diagnosis of HIV, who were therefore not using PcP prophylaxis. Though we did not collect data on preadmission antimicrobial use, most (98\%) patients presented with fever, and given the common use of sulfadoxine-pyrimethamine for presumptive antimalarial fever treatment in Uganda, many patients may have received incidental therapy for $\mathrm{PcP}$ with this medication prior to presentation. Finally, the low prevalence may be the result of complex local ecological and host factors which have produced diverse rates of colonization in other locales. Given the emerging correlation between the local prevalence of colonization and the local incidence of disease, understanding the factors accounting for the epidemiology of colonization may inform understanding of clinical disease.

The low prevalence of colonization in the high-risk patients that we investigated supports the hypothesis that PcP results from human-to-human spread of $P$. jirovecii and not the reactivation of latent infection. Similar to populations in Europe and the United States, early life exposure to $P$. jirovecii is common in African populations. Given these data, incident $\mathrm{PcP}$ that resulted from reactivation would likely require prevalent colonization, particularly in patients that were heavily immunosuppressed and thus at high risk for PcP. That high-risk patients are infrequently colonized with $P$. jirovecii suggests that incident $\mathrm{PcP}$ in these patients more likely results from interpersonal spread. Furthermore, infrequent carriage in highly-immunosuppressed individuals also suggests that the population reservoir of latent organism for transmission is very limited. Taken together, these observations may account for the observed low incidence of PcP in HIV-infected patients in sub-Saharan Africa (van Oosterhout, Laufer et al. 2007; Kyeyune, den Boon et al. 2010) compared with Europe and the US.

Two other findings merit attention. First, we observed only two different mtLSUrRNA genotypes in the colonized patients, and three of seven patients had mixed genotypes. mtLSUrRNA genotypes have not been associated with disease severity and are more useful as a tool to better understand the epidemiology of $P$. jirovecii. The small number of isolates precludes significant conclusions, and the absence of genotypes 2 and 4 may simply result from chance.

Second, despite comparable degrees of immunosuppression, spectra of diagnoses, and patient demographics, a significantly greater proportion of $P$. jirovecii-colonized than noncolonized patients died soon after discharge. We believe that these patients were colonized and not infected because 1) the results of the Giemsa-stained slides of BAL were independently confirmed by a second technologist, and 2) a low organism count is suggested by negative results on both modified Giemsa staining and in PCR assays targeting both the single-copy $P$. jirovecii dihydropteroate synthase (dhps) and dihydrofolate reductase $(d h f r)$ genes (Huang, Taylor et al. 2010). However, we cannot exclude the possibility that these colonized patients subsequently developed PcP and died from this disease. Nevertheless, the limited number of colonized patients precludes a comprehensive multivariate analysis of risk factors for death, and this preliminary bivariate association should be explored in future studies before any causal relationship is inferred. 
Our study has several limitations. Though the nested PCR assay we used is considered highly sensitive for $P$. jirovecii, more intensive specimen collection may increase yield (Ponce, Gallo et al. 2010). Nevertheless, the use of this assay enables comparison with other studies from different settings that have documented much higher prevalences of colonization. The high frequency of reported use of PcP prophylaxis among those with known HIV infection may have decreased the prevalence of colonization. Nevertheless, as noted, prevalence was also low among those not taking prophylaxis. Finally, though we note a bivariate association between colonization and death after admission, a comprehensive analysis of risk factors for death is impossible given the limited number of colonized patients, as above.

In this cross-sectional study of HIV-infected patients in Kampala with pneumonia that employed aggressive diagnostic measures, the prevalence of $P$. jirovecii colonization was low. Our study informs the understanding of the epidemiology of $P$. jirovecii in HIVinfected patients in Uganda, where HIV infection is prevalent. Further studies utilizing lessinvasive oropharyngeal and sputum specimens and molecular testing can characterize incident and prevalent $P$. jirovecii infection in African populations at greatest risk of clinically-significant infection.

\section{Acknowledgments}

\section{Funding}

This work was supported by the National Heart, Lung, and Blood Institute of the National Institutes of Health [K24 HL087713 and R01 HL090355-04 to L.H.] and by the National Institute of Allergy and Infectious Diseases [K23 AI080147 to J.L.D.].

We thank John Kiidha and Patrick Byanyima for their efforts in the MIND/IHOP laboratory in Mulago Hospital, the administration of Mulago Hospital for their support of our research and patient care efforts, and Florence Nankya and Rachael Kyeyune for their care of the patients. Ultimately, we are indebted to the patients who participated in the study.

\section{References}

Batungwanayo J, Taelman H, et al. Pulmonary disease associated with the human immunodeficiency virus in Kigali, Rwanda. A fiberoptic bronchoscopic study of 111 cases of undetermined etiology. Am J Respir Crit Care Med. 1994; 149(6):1591-6. [PubMed: 8004318]

Beard CB, Carter JL, et al. Genetic variation in Pneumocystis carinii isolates from different geographic regions: implications for transmission. Emerg Infect Dis. 2000; 6(3):265-72. [PubMed: 10827116]

Daly KR, Fichtenbaum CJ, et al. Serologic responses to epitopes of the major surface glycoprotein of Pneumocystis jiroveci differ in human immunodeficiency virus-infected and uninfected persons. J Infect Dis. 2002; 186(5):644-51. [PubMed: 12195351]

Davis JL, Welsh DA, et al. Pneumocystis colonisation is common among hospitalised HIV infected patients with non-Pneumocystis pneumonia. Thorax. 2008; 63(4):329-34. [PubMed: 18024536]

Huang L, Crothers K, et al. Pneumocystis colonization in HIV-infected patients. J Eukaryot Microbiol. 2003; 50(Suppl):616-7. [PubMed: 14736184]

Huang L, Taylor SM, et al. Low prevalence of Pneumocystis pneumonia but high prevalence of Pneumocystis dihydropteroate synthase gene mutations in HIV-infected persons in Uganda. Am J Respir Crit Care Med. 2010; 181:A5212.

Kyeyune R, den Boon S, et al. Causes of early mortality in HIV-infected TB suspects in an East African referral hospital. J Acquir Immune Defic Syndr. 2010; 55(4):446-50. [PubMed: 21105258]

Madhi SA, Cutland C, et al. Ineffectiveness of trimethoprim-sulfamethoxazole prophylaxis and the importance of bacterial and viral coinfections in African children with Pneumocystis carinii pneumonia. Clin Infect Dis. 2002; 35(9):1120-6. [PubMed: 12384847] 
Miller RF, Lindley AR, et al. Genotypic variation in Pneumocystis jirovecii isolates in Britain. Thorax. 2005; 60(8):679-82. [PubMed: 16061710]

Morris A, Kingsley LA, et al. Prevalence and clinical predictors of Pneumocystis colonization among HIV-infected men. AIDS. 2004; 18(5):793-8. [PubMed: 15075515]

Morris A, Lundgren JD, et al. Current epidemiology of Pneumocystis pneumonia. Emerg Infect Dis. 2004; 10(10):1713-20. [PubMed: 15504255]

Morris A, Wei K, et al. Epidemiology and clinical significance of pneumocystis colonization. The Journal of infectious diseases. 2008; 197(1):10-17. [PubMed: 18171279]

Ndyomugyenyi R, Magnussen P, et al. Diagnosis and treatment of malaria in peripheral health facilities in Uganda: findings from an area of low transmission in south-western Uganda. Malaria Journal. 2007; 6:39. [PubMed: 17407555]

Nkinin SW, Daly KR, et al. Evidence for high prevalence of Pneumocystis jirovecii exposure among Cameroonians. Acta Trop. 2009; 112(2):219-24. [PubMed: 19665440]

Ponce CA, Gallo M, et al. Pneumocystis colonization is highly prevalent in the autopsied lungs of the general population. Clin Infect Dis. 2010; 50(3):347-53. [PubMed: 20047487]

van Oosterhout JJ, Laufer MK, et al. Pneumocystis pneumonia in HIV-positive adults, Malawi. Emerging infectious diseases. 2007; 13(2):325-328. [PubMed: 17479904]

Wakefield AE. DNA sequences identical to Pneumocystis carinii f. sp. carinii and Pneumocystis carinii f. sp. hominis in samples of air spora. J Clin Microbiol. 1996; 34(7):1754-9. [PubMed: 8784583]

Wakefield AE, Lindley AR, et al. Limited asymptomatic carriage of Pneumocystis jiroveci in human immunodeficiency virus-infected patients. J Infect Dis. 2003; 187(6):901-8. [PubMed: 12660936]

Wakefield AE, Stewart TJ, et al. Infection with Pneumocystis carinii is prevalent in healthy Gambian children. Trans R Soc Trop Med Hyg. 1990; 84(6):800-2. [PubMed: 2096511]

Worodria W, Okot-Nwang M, et al. Causes of lower respiratory infection in HIV-infected Ugandan adults who are sputum AFB smear-negative. Int J Tuberc Lung Dis. 2003; 7(2):117-23. [PubMed: 12588011] 
Table 1

Characteristics of colonized and non-colonized patients

\begin{tabular}{|c|c|c|c|c|}
\hline & \multicolumn{4}{|c|}{ Colonized } \\
\hline & Total $(n=124)$ & Yes $(n=7)$ & No $(n=117)$ & p-value ${ }^{a}$ \\
\hline \multicolumn{5}{|l|}{ Presenting characteristics } \\
\hline Age, years, median (IQR) & $33(28-39)$ & $38(27-44)$ & $32(28-38)$ & 0.57 \\
\hline Women, \% (no.) & $57(71)$ & $57(4)$ & $57(67)$ & 1.0 \\
\hline \multicolumn{5}{|l|}{ Final diagnosis ${ }^{b}, \%$ (no.) } \\
\hline Tuberculosis & $30(37)$ & $43(3)$ & $29(34)$ & \multirow{4}{*}{0.36} \\
\hline Bacterial pneumonia & $23(29)$ & 0 & $25(29)$ & \\
\hline Multiple diagnoses & $24(30)$ & $43(3)$ & $23(27)$ & \\
\hline Unknown & $23(28)$ & $14(1)$ & $23(27)$ & \\
\hline New diagnosis of HIV, \% (no.) & $25(31)$ & $29(2)$ & $25(29)$ & 1.0 \\
\hline Known HIV, \% (no.) & $75(93)$ & $71(5)$ & $75(88)$ & 1.0 \\
\hline CD4 cells/mm³ $\mathbf{m m}^{3}$ median (IQR) & $88(22-196)$ & $58(9-124)$ & $91(23-211)$ & 0.48 \\
\hline PcP prophylaxis on admission ${ }^{c}, \%$ (no.) & $83(77)$ & $100(5)$ & $82(72)$ & 0.58 \\
\hline ARVs on admission $c, \%$ (no.) & $24(22)$ & $20(1)$ & $21(24)$ & 1 \\
\hline \multicolumn{5}{|l|}{ Outcome } \\
\hline Died within 2 months following discharge, \% (no.) & $27(34)$ & $71(5)$ & $25(29)$ & 0.016 \\
\hline
\end{tabular}

HIV: human immunodeficiency virus. ARV: antiretroviral

${ }^{a}$ Determined using Fisher's exact or the Kruskal-Wallis test.

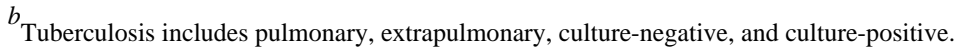

Multiple diagnoses includes those listed as well as Cryptococcus neoformans. Final diagnosis was categorized as "unknown" when the patient died prior to completing the diagnostic evaluation or when the evaluation was negative and any response to empiric therapy was unable to be assessed because the patient was lost to follow-up.

${ }^{c}$ Among 93 patients with known HIV infection. 